\title{
Unterschiedliche Pflege todkranker Krebspatienten am Lebensende
}

\section{Hier steht eine Anzeige.}

In einer Kohortenstudie fanden sich geschlechtsspezifische Unterschiede in der Pflege von Krebspatienten in ihrer letzten Lebensphase. Eine empathische, aber realistische Beratung kann die Entscheidung gegen eine aggressive EoL-Pflege beeinflussen.

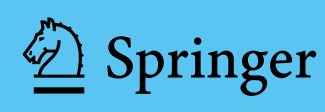

$\mathrm{V}$ iele Krebspatienten erhalten am Ende des Lebens (End of Life, EoL) trotz des absehbaren Todes eine aggressive Behandlung mit Einweisung auf eine Intensivstation und Beginn einer neuen Chemotherapie. Selten wird dadurch die Lebenszeit verlängert oder die Lebensqualität verbessert. Ein zentraler Punkt für die Entscheidung für eine solche Pflege ist die Kommunikation zwischen Ärzten, Patienten und Angehörigen, in denen Prognose, Ziele und Wertvorstellungen der Patienten einfließen sollten. Kleinere Studien zeigen, dass es geschlechtspezifische Unterschiede in der Einstellung zu Sterben und Tod sowie dem Verhalten in dieser Lebensphase gibt - Frauen entscheiden sich eher gegen eine aggressive Pflege als Männer.

Eine prospektive, multizentrische Kohortenstudie wollte die Unterschiede näher beleuchten. Dazu wurden die Daten von 353 Patienten mit metastasiertem Krebs im Endstadium herangezogen, die im Median 4,1 Monate vor ihrem Tod an einer Befragung mit Schwerpunkt auf möglichen Prädiktoren für die EoL-Pflege teilgenommen hatten und für die Angaben zur Art der Pflege kurz vor ihrem Tod vorlagen. Ergebnis: Frauen lehnten häufiger als Männer Maßnahmen zur Wiederbelebung ab ( $48 \%$ vs. $37 \%, \mathrm{p}=0,039)$ und bevorzugten eine palliative EoL-Pflege ( $70 \%$ vs. $58 \%, \mathrm{p}=0,017$ ). Umgekehrt waren Patienten, die am Lebensende eine intensivmedizinische Behandlung erhielten, eher männlich ( $73 \%$ vs. $52 \%, \mathrm{p}=0,015)$ und lehnten eine palliativmedizinische Behandlung ab ( $35 \%$ vs. $67 \%, \mathrm{p}<0,001)$.

Nach Adjustierung auf mögliche Confounder verbrachten Männer, die vorher eine Beratung zur EoL-Pflege erhalten hatten, weniger häufig ihre letzte Lebenswoche auf einer Intensivstation als Männer, die nicht beraten worden waren (adjustierte Odds ratio 0,26, $95 \%$-Konfidenzintervall 0,07-0,91; $p=0,04)$. Bei Frauen bestand kein Unterschied zwischen denen, die beraten wurden oder keine Beratung erhielten.

Fazit: Männer im finalen Krebsstadium erhalten öfter als Frauen eine intensivmedizinische Behandlung, die die Lebensqualität mindert und die Lebenspanne kaum verlängert. Eine eingehende Beratung der Männer senkt den Wunsch nach aggressiver Therapie am Lebensende. Bei Frauen besteht auch ohne Beratung die Tendenz zu einer eher palliativen EoL-Pflege.

Barbara Kreutzkamp

Sharma RK et al. Male-female patient differences in the association between end-of-life discussions and receipt of intensive care near death.

Cancer. 2015; 121 (16): 2814-20 